\title{
BMJ Open Indigenous health worker support for patients with poorly controlled type 2 diabetes: study protocol for a cluster randomised controlled trial of the Mana Tù programme
}

\author{
Vanessa Selak, ${ }^{1}$ Tereki Stewart, ${ }^{2}$ Yannan Jiang, ${ }^{3}$ Jennifer Reid, ${ }^{4}$ Taria Tane ${ }^{2}$ \\ Peter Carswell, ${ }^{5}$ Matire Harwood ${ }^{4}$
}

To cite: Selak V, Stewart T, Jiang $Y$, et al. Indigenous health worker support for patients with poorly controlled type 2 diabetes: study protocol for a cluster randomised controlled trial of the Mana Tū programme. BMJ Open 2018;8:e019572. doi:10.1136/ bmjopen-2017-019572

- Prepublication history and additional material for this paper are available online. To view these files, please visit the journal online (http://dx.doi org/10.1136/bmjopen-2017019572).

Received 15 March 2018 Revised 9 July 2018 Accepted 28 September 2018

Check for updates

(C) Author(s) (or their employer(s)) 2018. Re-use permitted under CC BY-NC. No commercial re-use. See rights and permissions. Published by BMJ.

For numbered affiliations see end of article.

Correspondence to

Dr Matire Harwood;

m.harwood@auckland.ac.nz

\section{ABSTRACT}

Introduction Type 2 diabetes mellitus (T2DM) and its complications are more common among Māori and Pacific people compared with other ethnic groups in New Zealand. Comprehensive and sustained approaches that address social determinants of health are required to address this condition, including culturally specific interventions. Currently, New Zealand has no comprehensive T2DM management programme for Māori or Pacific people. Methods and analysis The Mana Tū programme was developed by a Māori-led collaborative of primary healthcare workers and researchers, and codesigned with whānau (patients and their families) in order to address this gap. The programme is based in primary care and has three major components: a Network hub, Kai Manaaki (skilled case managers who work with whānau with poorly controlled diabetes) and a cross-sector network of services to whom whānau can be referred to address the wider determinants of health. The Network hub supports the delivery of the intervention through training of Kai Manaaki, referrals management, cross-sector network development and quality improvement of the programme. A two-arm cluster randomised controlled trial will be conducted to evaluate the effectiveness of the Mana Tū programme among Māori, Pacific people or those living in areas of high socioeconomic deprivation who also have poorly controlled diabetes (glycated haemoglobin, HbA1c, $>65 \mathrm{mmol} / \mathrm{mol}(8 \%))$, compared with being on a wait list for the programme. A total of 400 participants will be included from 10 general practices ( 5 practices per group, 40 participants per practice). The primary outcome is HbA1C at 12 months. Secondary outcomes include blood pressure, lipid levels, body mass index and smoking status at 12 months. This protocol outlines the proposed study design and analysis methods.

Ethics and dissemination Ethical approval for the trial has been obtained from the New Zealand Health and Disability Ethics Committee (17/NTB/249). Findings will be presented to practices and their patients at appropriate fora, and disseminated widely through peer-reviewed publications and conference presentations.

Trial registration number ACTRN12617001276347; Preresult.

\section{Strengths and limitations of this study}

- Mana Tū is the first comprehensive programme for Māori with type 2 diabetes mellitus.

- The programme was developed by a Māori-led collaborative of primary healthcare workers and researchers, and codesigned with whānau (patients and their families).

- Mana Tū is based in primary care and has three major components: a Network hub, Kai Manaaki (skilled case managers who work with whānau with poorly controlled diabetes) and a cross-sector network of services to whom whānau can be referred to address the wider determinants of health.

- The effectiveness of the intervention compared with being on a wait list for the programme will be assessed in a cluster randomised controlled trial.

- This is a pragmatic trial and outcome data (including the primary outcome, glycated haemoglobin at 12 months) will be obtained from routinely collected electronic data.

\section{INTRODUCTION}

Type 2 diabetes mellitus (T2DM) and its complications are more common among Māori, the indigenous people and Pacific people compared with other ethnic groups in New Zealand. ${ }^{1}$ Among adults, the prevalence of self-reported diabetes in New Zealand is $9.0 \%$ (95\% CI $7.7 \%$ to $10.5 \%$ ) in Māori, $13.9 \%$ (95\% CI $10.9 \%$ to $17.5 \%$ ) in Pacific, $6.4 \%(95 \%$ CI $4.9 \%$ to $8.3 \%)$ in Asian and $5.3 \%$ (95\% CI $4.8 \%$ to $5.9 \%$ ) in European/ other people. ${ }^{2}$ Among people aged 15 years or older, the rate of renal failure with concurrent diabetes is 97.1 (95\% CI 91.7 to 102.7) per 100000 in Māori and 17.5 per 100000 in non-Māori and the rate of lower limb amputation with concurrent diabetes is 31.4 (95\% CI 28.4 to 34.6) per 100000 in Māori and 9.1 (95\% CI 8.7 to 9.6$)$ per 100000 in non-Māori. ${ }^{3}$ 
Deaths due to diabetes in Pacific people are estimated to be more than nine times the rate of those in non-Māori, non-Pacific people in Auckland, in which $71 \%$ of Pacific people living in New Zealand reside. ${ }^{4}$ Comprehensive and sustained approaches that address social determinants of health (such as income and housing) are required to address this condition, ${ }^{1}$ including culturally specific interventions. ${ }^{5}$ Currently, New Zealand has no comprehensive T2DM management programme for Māori or Pacific people. ${ }^{6}$

The Oranga Ki Tua Advisory Roopu, a Māori-led collaborative, was convened by the National Hauora Coalition (NHC), a Māori-led Primary Health Organisation (PHO) with 33 affiliated general practices, in order to develop an evidence-based programme for whānau (patients and their families) in order to address this gap. The Roopu included whānau, clinicians, researchers, health service planners and providers of whānau ora services (these are cross-sector services that focus on improving the well-being of families, not just individuals). Mana Tū (meaning 'to stand with authority') is a programme founded on the recommendations from the Roopu and specifically seeks to address system (Māori leadership, health equity, wider determinants), service (integrated primary care) and patient (taking charge, whānau ora, the journey) factors that impact on the whānau's ability to 'mana tū'. A framework for change was developed which brings all three levels (patient, service, system) together (figure 1).

The Mana Tū programme is based in primary care and has three major components: a Network hub, Kai Manaaki (skilled case managers who work with whānau with poorly controlled diabetes) and a cross-sector network of services to whom whānau can be referred to address the wider determinants of health. The Network hub supports the delivery of the intervention through training of Kai Manaaki, referrals management, crosssector network development and quality improvement of the programme.

Evaluation of the Mana Tū programme, guided by kaupapa Māori research methodology and methods (ie, an approach based on critical theory and Māori ideology, principles and practice), will use a range of quantitative and qualitative methods to address a number of research questions. This paper describes the protocol of one aspect of the evaluation, a cluster randomised controlled trial. This trial is proposed to determine the effectiveness of the Mana Tū programme in improving glycaemic control (glycated haemoglobin, HbA1c) among people with

\begin{tabular}{|c|c|c|c|c|}
\hline \multicolumn{5}{|c|}{$\begin{array}{l}\text { Programme goal: To support general practice to establish an environment in which discrimination is addressed in order for patients and whānau engage in improving their health and } \\
\text { social outcomes. }\end{array}$} \\
\hline \multicolumn{4}{|c|}{$\begin{array}{l}\text { Theory of change: } \\
\text { - The Kai Manaaki will support the patients and whānau to determine goals and access services to achieve those goals. } \\
\text { - } \quad \text { Addressing institutional discrimination within the health system will help improve patient engagement and trust in the } \\
\text { - } \quad \text { Support is required to help general practices support their patients in improving social outcomes } \\
\text { - } \quad \text { Improving engagement with the practice will improve diabetes self-management } \\
\text { - Building linkages across health and social agencies will support patient engagement } \\
\text { Building an evidence base for Mana Tú will support sustainable funding }\end{array}$} & \multirow{3}{*}{ 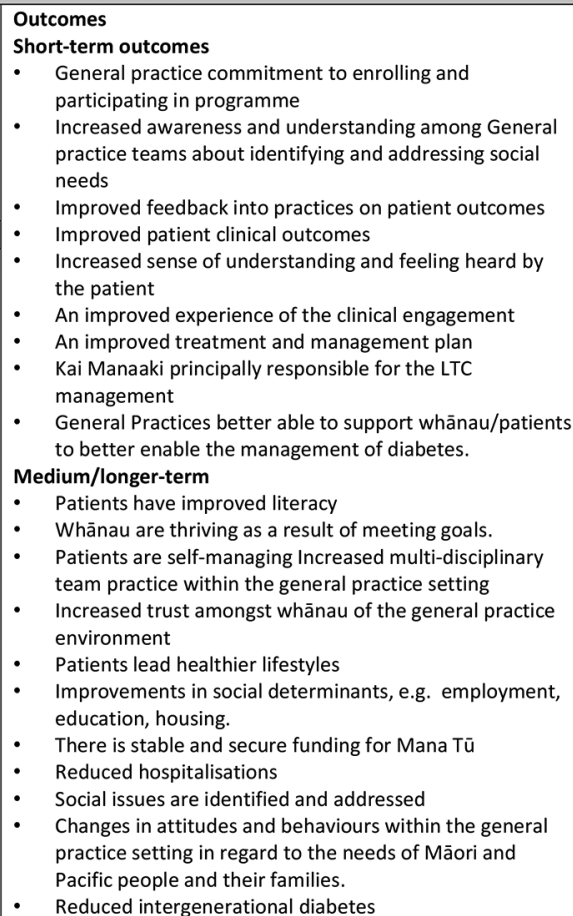 } \\
\hline Context and need & Inputs & Activities & Outputs & \\
\hline $\begin{array}{l}\text { There are poor } \\
\text { outcomes for Māori } \\
\text { and Pacific peoples } \\
\text { There is a variable } \\
\text { quality of care, with } \\
\text { social issues often not } \\
\text { addressed } \\
\text { There is systemic } \\
\text { institutional racism } \\
\text { within the health } \\
\text { system } \\
\text { Many Māori and Pacific } \\
\text { peoples distrust the } \\
\text { health system } \\
\text { There is a mismatch } \\
\text { between clinical } \\
\text { services and the } \\
\text { person's needs } \\
\text { There is not a } \\
\text { commitment to } \\
\text { sustainable funding for } \\
\text { innovative approaches } \\
\text { to LTC management }\end{array}$ & 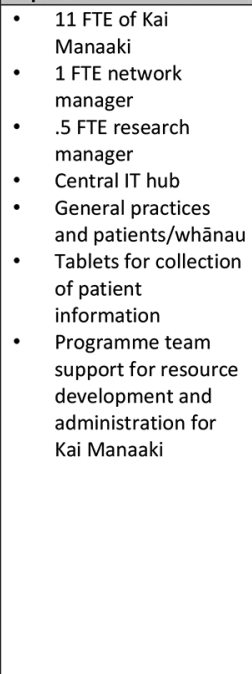 & 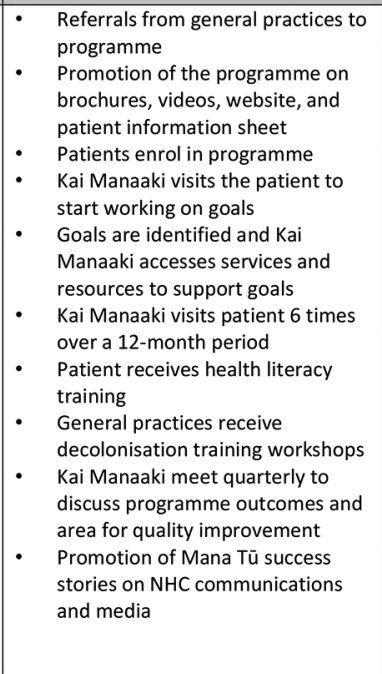 & 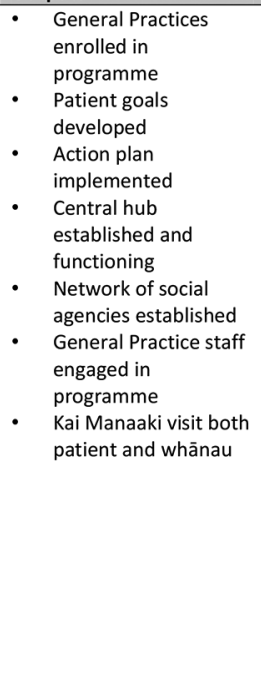 & \\
\hline \multicolumn{3}{|l|}{ Enablers } & \multicolumn{2}{|l|}{ Challenges } \\
\hline \multicolumn{3}{|c|}{$\begin{array}{l}\text { Clinical champion in practices } \\
\text { Whānau champions } \\
\text { There is a number of funding channels available } \\
\text { NHC has a track record of designing programmes that are effective } \\
\text { A focus on whānau moko }\end{array}$} & \multicolumn{2}{|c|}{$\begin{array}{l}\text { - There is an obesogenic environment } \\
\text { - There is a near total reliance on Kai Manaaki as relationship brokers } \\
\text { - } \quad \text { need to address the wider social determinants of health } \\
\text { - Limited understanding on what drives the range of social agencies that Mana Tū } \\
\text { needs to work with } \\
\text { - There is turnover of staff in social agencies, which creates a need to be renewing } \\
\text { relationships } \\
\text { - A focus on mental health is currently lacking in the Mana Tū design }\end{array}$} \\
\hline
\end{tabular}

Figure 1 Mana Tū framework for change. NHC, National Hauora Coalition. 


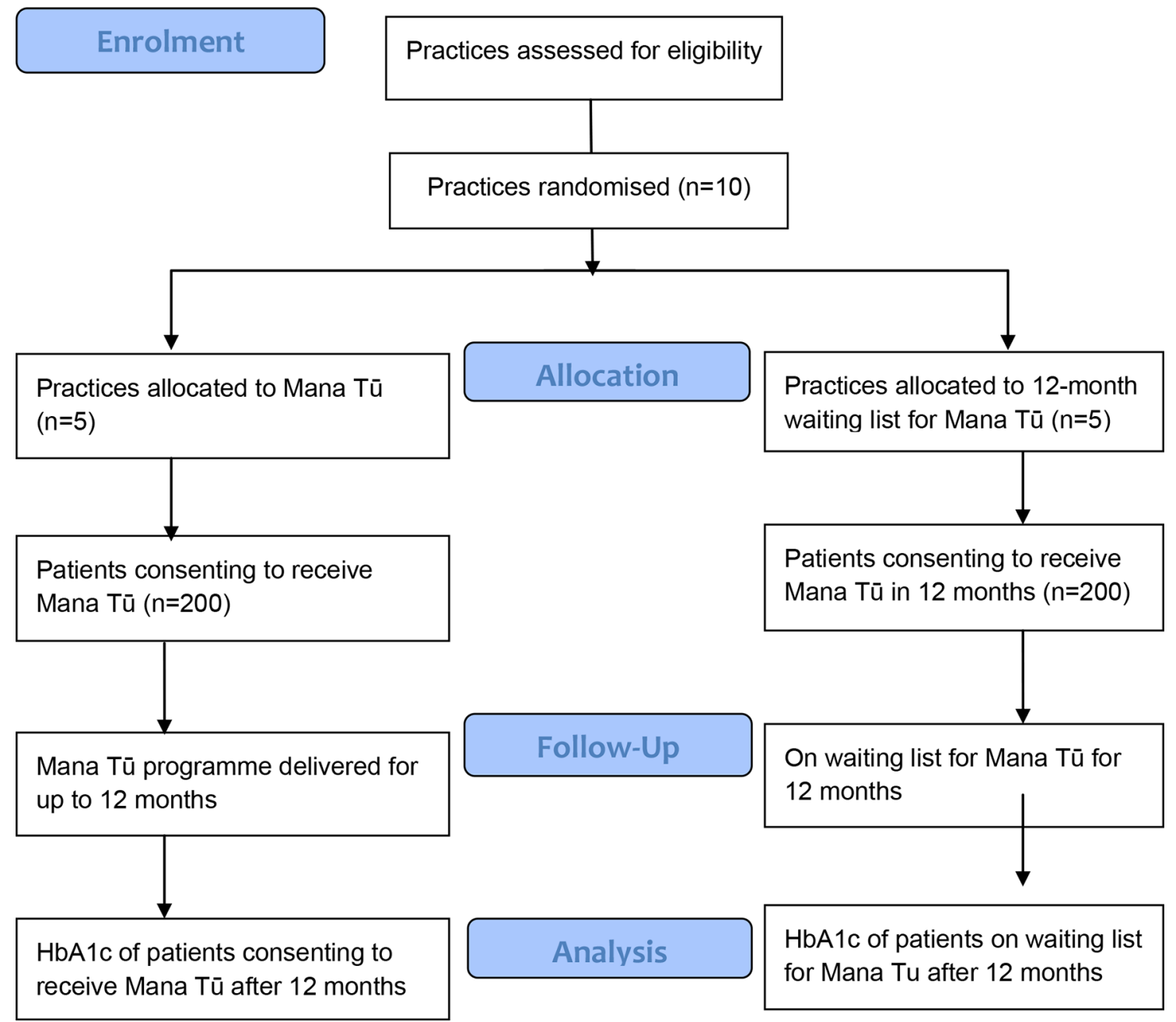

Figure 2 Trial flow diagram. HbA1c, glycated haemoglobin.

poorly controlled diabetes, in comparison with being on a 12-month wait list for the programme. This paper describes the design and protocol of the trial (V.2.0, dated 15 March 2018), consistent with the requirements of the Standard Protocol Items: Recommendations for Interventional Trials 2013 Statement. ${ }^{7}$

\section{METHODS AND ANALYSIS \\ Objective}

The primary objective is to determine the effectiveness of Mana Tū programme compared with being on a 12-month wait list for the programme on HbAlc at 12 months. Secondary objectives are to determine the effectiveness of Mana Tū compared with being on a 12-month wait list for the programme on blood pressure, lipid levels, body mass index and smoking status at 12 months.

\section{Trial design}

A two-arm, parallel, cluster randomised controlled trial will be conducted (figure 2). A cluster trial design was selected, with practice as the unit of randomisation, to minimise the risk of contamination between patients treated in the same general practice and to enhance the feasibility of programme delivery. The trial was initiated in March 2018, recruitment is expected to continue until December 2018 and follow-up is expected to be completed by December 2019.

\section{Study setting}

The Mana Tū programme will be implemented in general practices affiliated with the NHC, a Māori-led PHO (https://www.nhc.maori.nz/clinic-network). PHOs are groups of providers working with their communities to improve health and reduce health inequities by coordinating and providing essential primary healthcare services, including general practice services, to an enrolled population in New Zealand. ${ }^{8}$ The NHC will act as the Network hub for the Mana Tū programme, and will communicate important protocol modifications to relevant parties. The study will be set in the practices affiliated with the NHC, which are predominately based in the Auckland region of New Zealand, because it will use routinely collected electronic data and such data are already provided to the NHC by these practices using established and secure methods that protect patient confidentiality. 


\section{General practice eligibility}

NHC practices will be contacted and their consent requested for participation in the trial. Practices will be eligible for inclusion in the trial if they meet all four practice inclusion criteria: (1) agree to support the implementation of the Mana Tū programme in their practice; (2) agree to review and confirm trial eligibility for each of their potentially eligible patients; (3) agree to arrange HbAlc testing at baseline and 12 months if not already available in the electronic medical record. Practices will be approached in descending order according to their number of potentially eligible patients, until there are a sufficient number of practices enrolled in the trial.

\section{Patient eligibility}

Potentially eligible patients will be identified centrally using data from electronic practice records that are already provided to the NHC. Each participating practice will review the eligibility of their patients for the trial, and will refer all eligible patients to the Mana Tū programme Network hub (NHC).

Patients are eligible for the trial if all of the following inclusion criteria are met: (1) most recent HbAlc (no more than 3 months prior to the trial start date for the practice) of $65 \mathrm{mmol} / \mathrm{mol}(8 \%)$ or more ${ }^{9}$ (2) aged at least 18 years; (3) self-identified prioritised ethnicity Māori or Pacific (as defined by New Zealand ethnicity data protocols ${ }^{10}$ ) or living in an area with high socioeconomic deprivation (quintile 5 of the New Zealand Index of Deprivation $\left.^{11}\right)$; (4) nothing known to the practice about the patient that would preclude them from being able to participate in the intervention or that would make participation in the intervention inappropriate (eg, unable to consent to receiving the Mana Tū programme, terminal illness, planning to move overseas); (5) the patient has been referred to the Mana Tū programme Network hub (NHC) and (6) the patient has provided written informed consent for participation in the trial.

\section{Randomisation and blinding}

The randomisation units will be 10 general practices. For the purpose of stratification, each practice will be matched to another similar practice according to three key factors: location (rural vs urban), District Health Board (DHB, as each DHB has its own set of diabetes services delivered in primary care such as nurse-led clinics) and structure of the clinic (whether or not the practice is marae based, as marae-based practices already have associated social services).

Practices will be randomised at a 1:1 ratio to receive either the Mana Tū programme or a 12-month wait list for the programme. The matched practice will receive the alternative treatment for comparison. The randomisation schedule will be computer generated and prepared by the Network hub before treatment allocation.

It is not possible to blind participants or healthcare providers due to the nature of the intervention, however, objective outcomes (HbA1c) will be measured by community laboratories with no knowledge of trial participation or treatment allocation, and data from community laboratories (including HbA1c) is sent electronically and entered automatically into the electronic medical record. Statistical analysis will be conducted postdata lock after all trial data have been collected.

\section{Participant consent}

After the practice has been randomised (irrespective of the treatment arm), the Network hub will assign a Kai Manaaki to the practice. The Kai Manaaki will approach all eligible patients referred to the Network hub, to discuss the trial and Mana Tù with them. Eligible patients who provide written informed consent to participation in the trial and the Mana Tū programme (either delivered then or in 12 months time) will be included in the trial. Once the Kai Manaaki has identified 40 trial participants in the practice, no further participants will be sought from that practice to ensure that the case load of the Kai Manaaki is manageable.

\section{Intervention and control arm}

The Mana Tū programme comprises three key components: a Network hub (NHC), Kai Manaaki (skilled case managers who work with whānau with poorly controlled diabetes) and a cross-sector network of services to whom whānau can be referred to address the wider determinants of health.

The Network hub (figure 3) will support the delivery of the Mana Tū programme to primary care practices. The hub will have a critical organising function which supports the delivery of the intervention across multiple providers. The hub will support service performance, continued learning and ongoing development. Key functions will include: training on the Mana Tū intervention model including standardised guidelines and protocols; data-driven quality and process improvement activity as part of a learning network; network development including alliancing with range of health and social agencies to support case management activity; and referrals management. One of the key enablers of the intervention will be a data system to support case management, quality improvement activity and performance management. NHC uses an existing information management platform (Mōhio) that is integrated within primary care patient management systems (including patient electronic medical records) as well as standalone to support effective case management. This will enable timely and standardised data capture of clinical and non-clinical information.

Kai Manaaki are skilled case managers supported by the hub. Kai Manaaki will undertake the activities outlined in table 1 with eligible patients in practices randomised to the intervention. Their case load will be restricted to 30 patients at any one time.

Cross-sector services will be accessed, via the Kai Manaaki and with the support of the Network hub, to ensure that broader determinants of health are addressed 


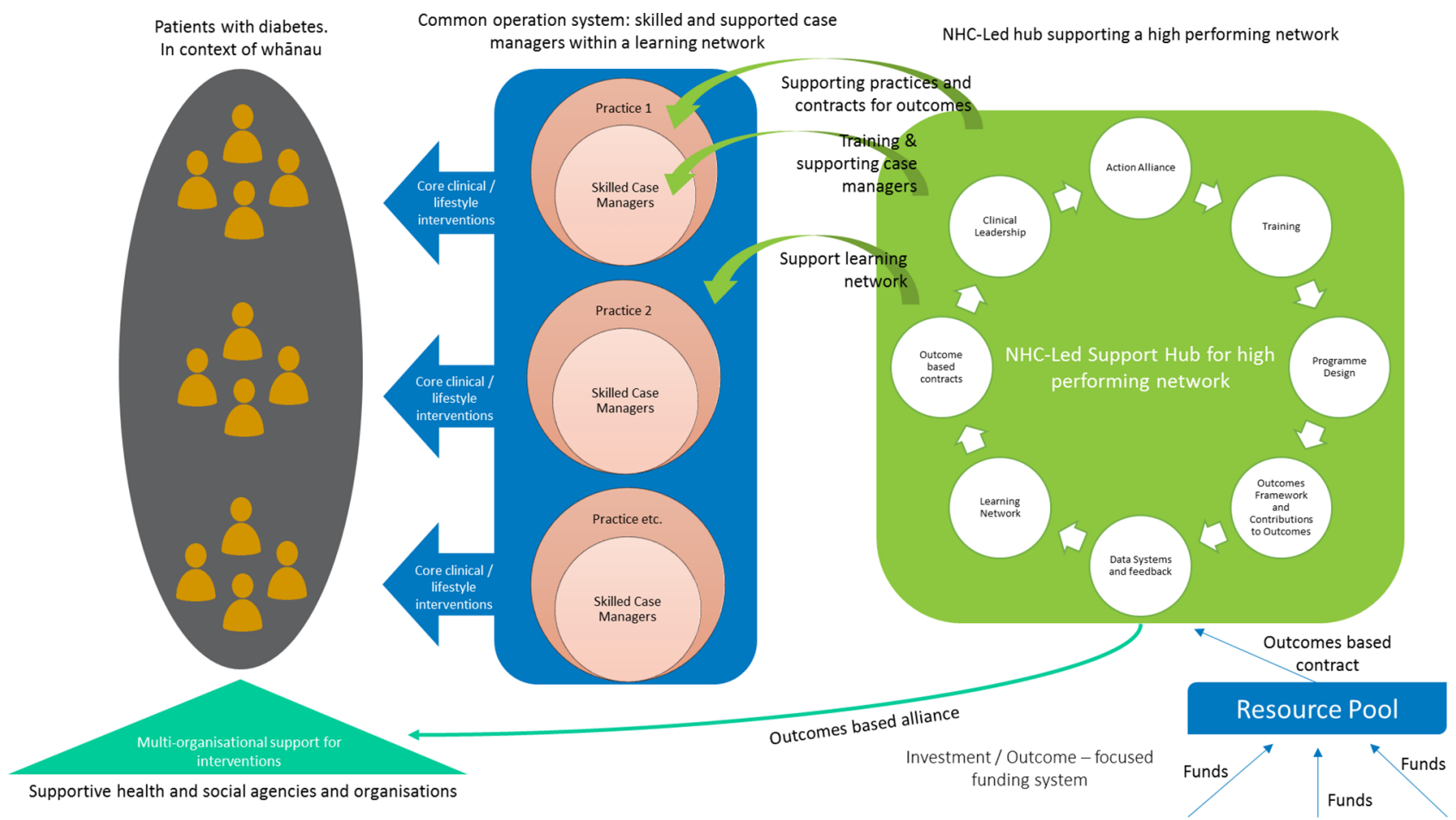

Figure 3 Network hub for Mana Tū programme. NHC, National Hauora Coalition.

for the person and/or their family. Therefore, Mana Tù has been designed to work at the multiple (system, service and patient/family) levels. The programme has been designed to be implemented in a series of pragmatic steps to support the person's journey across all three of these levels (table 1 , figure 4 ).

Irrespective of treatment allocation, both groups of practices will continue to provide usual care to all of their patients for the management of diabetes according to relevant guidelines, including regular monitoring of $\mathrm{HbAlc}$, blood pressure and lipid levels. ${ }^{12-14}$ Patients in practices randomised to the wait list control group will receive the Mana Tū programme after 12 months follow-up.

\section{Outcomes}

The primary outcome for the trial will be HbA1c at 12 months after the trial start date for the patient's general practice. Routinely collected HbAlc will be obtained from the practice electronic medical record. A 3-month window will be applied to the HbAlc measurement, before or after the scheduled assessment date at 12 months. Where multiple values are available within this time window, the nearest value prior to the 12-month end of trial date will be used; otherwise the nearest value after the 12-month end of trial date will be used.

Secondary outcomes will also be measured at 12 months: blood pressure, lipid levels, body mass index and smoking status. Mortality and acute hospitalisation rates during the 12-month follow-up period will also be compared between groups, along with secondary care resource utilisation.

The Mana Tū programme includes a range of initial assessments (online supplementary appendix), which will be monitored over time as part of the programme, but data from these assessments are not able to be collected from participants waiting for Mana Tū due to funding constraints, and therefore will not be considered as part of the trial. Data from these assessments will be used in other (concurrent) evaluations of the Mana Tù programme.

\section{Sample size}

We aim to recruit a total of 400 participants from 10 general practices (5 practices per group, 40 participants per practice). This sample size will provide $80 \%$ power at $5 \%$ significance level (two sided) to detect a betweengroup difference of $6 \mathrm{mmol} / \mathrm{mol}(0.5 \%)$ in HbAlc at 12 months, assuming an SD of $16 \mathrm{mmol} / \mathrm{mol}(1.5 \%)$ and an intracluster correlation coefficient (ICC) of 0.01. A recent meta-analysis of diabetes quality improvement trials found that in trials in which participants had baseline HbAlc of at least $8.0 \%$ (equivalent to $64 \mathrm{mmol} / \mathrm{mol}$ ), the mean reduction in $\mathrm{HbAlc}$ with the intervention compared with the control was $0.46 \%$ (equivalent to $5 \mathrm{mmol} / \mathrm{mol}$ ). ${ }^{15}$ This trial is therefore powered to detect a similar difference in $\mathrm{HbAlc}$ between treatment groups. Estimates of the number of potentially eligible patients within each of the 33 general practices in the PHO (to inform the number of general practices required and participants per practice), SD and intraclass correlation of HbA1c were based on a de-identified analysis of potentially eligible patients from the NHC. Our estimate of sample size has not accounted for loss to follow-up because we will only be using routinely collected electronic practice data to which the $\mathrm{PHO} /$ Network hub already has access. 
Table 1 Kai Manaaki (KM) activities

When Activity

Prepatient consent for participation in Mana Tū programme

Visit 2

Visit 3 and every fortnight

Monthly

$6-12$ months

Activity

- KM integrated with each general practice (GP) clinic randomised to the Mana Tū intervention.

- Hub clinical leadership team (CLT) provides to each practice randomised to the Mana Tū intervention a list of all of the people enrolled at that practice with confirmed eligibility for the trial.

- Primary care clinician from the GP clinic contacts every person on the list to discuss the Mana Tū programme with them and to invite them to participate in the Mana Tū programme.

- KM contacts potential participant and arranges first visit.

- At first visit (in home or clinic or other site), KM engage with person \pm whannau, using the 'hui process' or similarly safe clinical engagement process. ${ }^{23}$

- Informed consent for participation in Mana Tū is obtained.

- KM undertakes the Mana Tū Assessment with participants (a formal assessment of clinical and wider determinants relevant to the self-management of type 2 diabetes mellitus (T2DM), online supplementary appendix).

- KM meets with person \pm whānau to complete the Mana Tū Plan (a plan incorporating aspects of self-management designed for indigenous people with $\mathrm{T}_{2} \mathrm{DM}^{24}$ and life domains that impact on social determinants as identified in the Mana Tū Assessment) including:

- goal setting with the person \pm whānau for self-management of risk factors and long-term conditions (including T2DM).

- identification of patient \pm whānau circumstances that impact negatively on life domains (social determinants) and 'walk alongside' whanau to facilitate resolution of issues.

- identification of cross-sector organisations required to support person \pm whānau.

- KM works with participants and whānau to achieve goals based on the Mana Tū Plan. KM will be integrating relevant services to provide appropriate care (eg, health literacy, smoking cessation) into the participant's Mana Tū Plan.

- KM will contact participants \pm whānau either in person or by phone when delivering the intervention and to provide information or feedback. Progress is recorded in the person's Plan and the KM database which is shared with the person and their whānau.

- KM will meet regularly with the primary care team at the GP clinic and cross-sector organisations to provide updates and, as required, seek their input.

- KM has access to the Hub CLT for additional support as required.

- Full review of participant (including attainment of goals) with primary care team at the GP clinic and Hub CLT.

- At midpoint (6 months), a full meeting with participant and whānau to review progress and update plans.

- From 9 months on, KM to start planning the participant's discharge from Mana Tū.

- Once discharge appropriate, KM completes discharge plan with person \pm whānau, general practice and relevant cross-sector organisations. Note this may occur at a time between 9 (minimum) and 12 (maximum) months from enrolment.

- A final assessment (data collection) at 12 months.

\section{Baseline assessment}

Baseline data (including HbAlc) will be obtained from the practice electronic medical record. As noted, the Network hub will check (prior to practice treatment allocation) that the most recent $\mathrm{HbAlc}$ for each patient is $65 \mathrm{mmol} / \mathrm{mol}(8 \%)$ or more and obtained no more than 3 months prior to the planned trial start date for the practice. Other data (including demography, clinical characteristics and secondary outcomes) will also be obtained from the practice electronic medical record, and while the most recent value will be used, there will not be the requirement for the data to have been obtained no more than 3 months prior to the planned trial start date for the practice.

\section{Twelve-month assessment}

Twelve months after the trial has started in the practice, irrespective of treatment allocation, the Network hub will obtain HbA1c from the practice electronic medical record. The HbAlc nearest to and within 3 months prior 


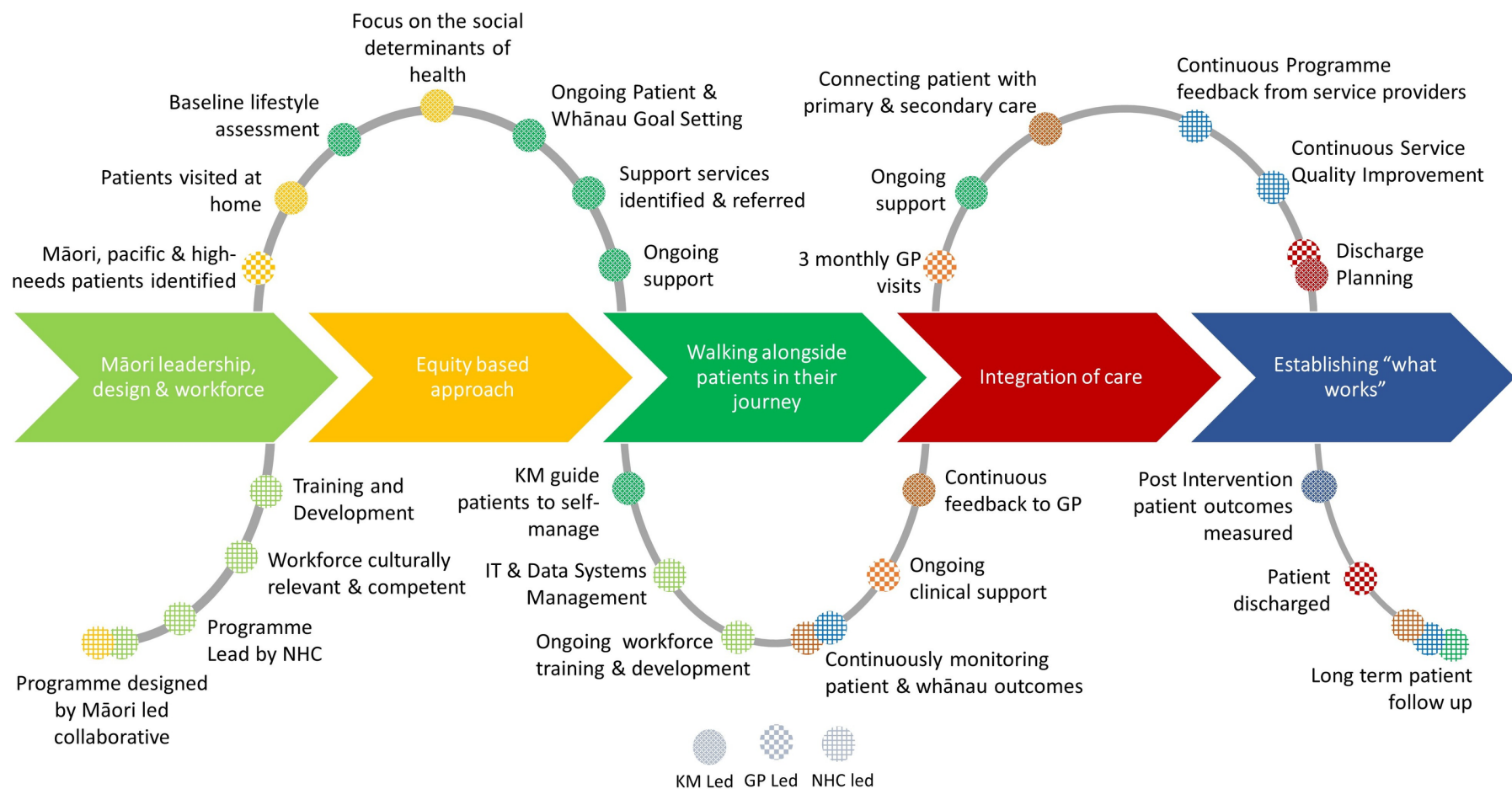

Figure 4 Mana Tū journey. GP, general practice; KM, Kai Manaaki; NHC, National Hauora Coalition.

to the 12-month end of trial date will be used; otherwise the $\mathrm{HbAlc}$ nearest to and within 3 months after the 12-month end of trial date will be used. If there is no such HbA1c value, the Network hub will request that the practice obtain this test from the patient within 3 months after the 12-month end of trial date.

Other follow-up data will also be obtained from the practice electronic medical record. The value nearest to and within 6 months prior to the 12-month end of trial date will be used; otherwise the value nearest to and within 3 months after the 12-month end of trial date will be used.

\section{Data management}

Trial data will be managed and its quality monitored by the Network hub, the NHC, which is the PHO for the eligible practices and already has access to practice data and established systems for data security and to protect patient confidentiality. Trial data will be extracted by the PHO for the trial statistician using unique (but non-identifiable) identifiers for practices and patients. A data monitoring committee is not required for the trial as all participants will continue to receive usual care for the management of diabetes, treatment allocation is unblinded and the intervention itself has a low risk of harm to participants.

\section{Statistical methods}

Data analysis will be performed using SAS V.9.4 (SAS). All statistical tests will be two sided at $5 \%$ significance level. The primary analysis will be conducted using intention-to-treat principles, including eligible participants recruited from all randomised general practices. Baseline demography and clinical characteristics of all individual participants will be presented by treatment group. Information collected at the level of general practices will also be described. Primary and secondary outcomes measured at 12 months will first be summarised using descriptive statistics. Continuous variables will be summarised as means and SDs. Categorical variables will be summarised as frequencies and percentages. The primary outcome (HbAlc at 12 months) will be analysed using generalised linear mixed model, adjusting for baseline HbAlc and other important confounders and accounting for the cluster effect of general practice. We will use routinely collected electronic patient data to which the $\mathrm{PHO} /$ Network hub already has access, therefore, the amount of loss to follow-up and missing data will be minimal at the individual level. Generalised linear mixed models use all data collected from the same clusters in maximum likelihood estimation, therefore, not only can this method of analysis control for correlated data between patients in the same cluster/practice using different covariance structures, but it can also take into account missing data at the same time. If the overall treatment effect is statistically significant in this superiority trial, further analyses will be undertaken to test the consistency of intervention effects across important subgroups (including age group, sex and smoking status, as well as categories of baseline HbA1c, blood pressure, lipids and body mass index). A similar approach will be applied to the secondary outcomes. ICCs will be estimated and reported. A statistical analysis plan will be developed a priori as the guide to final analysis post to data lock. Reporting of results will be in accordance with the principles of the Consolidated 
Standards of Reporting Trials statement extension to cluster randomised controlled trials. ${ }^{16}$

\section{Patient and public involvement}

The Mana Tū programme was codesigned with whānau (patients and their families) and strongly reflects the priorities, experience and preferences of Māori experiencing long-term conditions. The whole philosophy of the Mana Tū programme is to support whānau to 'mana tū' (ie, 'to stand with authority').

The overall research programme (including the trial) is informed and guided by kaupapa Māori research methodology and methods. In practice, this is demonstrated in the design of the intervention (by and for Māori whānau), by having Māori clinical and health research leadership in the project, and by using methods that place Māori at the centre of the research (eg, whānau inclusion, culturally safe practices, an intervention that addresses the broader determinants of health ${ }^{17}$ and a focus on systems issues rather than on individuals ${ }^{18}$ ). At a more fundamental level, the research fulfils a key principle of kaupapa Māori research methodology-tino rangatiratanga-in which Māori are allowed to control the ways in which we learn, understand and are empowered to create a future based on self-determined needs and aspirations. ${ }^{19}$ These themes are woven throughout the research design and methods.

The trial will only be using routinely collected electronic health data, thereby minimising participant burden, and these data will be accessed through the PHO to which patients already belong, using established systems for data security and to protect patient confidentiality.

The Oranga Ki Tua Advisory Roopu, including whānau, are being updated with trial progress and will be provided with trial results. Trial results will also be disseminated to trial participants at hui (meetings) organised by the NHC, along with Kai Manaaki in their direct interactions with whānau.

\section{DISCUSSION}

Despite experiencing a considerable burden of T2DM and its complications, no comprehensive T2DM management programmes have been developed for Māori or Pacific people. Mana Tū is the first such programme to be developed by Māori, for Māori. The programme was developed by a Māori-led collaborative of primary healthcare workers and researchers, and codesigned with whānau (patients and their families).

Now that this much needed intervention has been developed there is significant pressure to implement it without assessing its effectiveness using a randomised controlled trial. The randomised controlled trial is still the gold standard for evaluating an intervention, when properly designed, conducted and reported. ${ }^{20}$ This is because when interventions are allocated at random, there is a greater degree of assurance regarding the validity of a result than any observational study design. ${ }^{21}$ The randomised controlled trial gives greater confidence that the relationship observed between an exposure (such as an intervention) and outcome might be causal. ${ }^{22}$

When the intervention is delivered at a group level (as in this case, at the level of the general practice), there is a considerable risk of contamination ("unintentional spill over of intervention effects from one treatment group to another'). ${ }^{16}$ In this situation, while it is still desirable to conduct a randomised controlled trial, it is preferable to randomise at the level of the group/practice.

One of the major disadvantages of conducting randomised controlled trials is their cost-particularly for recruitment and data collection. This trial has been designed to leverage off, and to be as integrated as possible, with existing infrastructure, which will minimise the associated costs for recruitment and data collection. For example, once ethics approval has been obtained, potentially eligible patients can be identified by the $\mathrm{PHO} /$ Network hub, and, after they have consented to participating in the trial and receiving Mana Tù (either immediately or after a 12-month wait) their baseline and follow-up data can be obtained using routinely collected electronic data to which the $\mathrm{PHO} /$ Network hub already has access.

Another disadvantage of conducting a study with an inactive control arm is the lack of incentive for ongoing participation by those in the control group. This trial has been designed so that the inactive control arm is actually a waiting list for the intervention, which they will receive 12 months after the trial start date. This is likely to make the trial much more acceptable to practices and to encourage retention to the end of the trial for all practices irrespective of treatment allocation.

At the same time as the cluster randomised controlled trial, the Mana Tū Programme will also be evaluated using four additional studies, some of which will use data collected as part of the assessments included within the Mana Tū Programme (online supplementary appendix) One of these studies will investigate the cost-effectiveness of Mana Tū. The other three studies are qualitative: one will explore how the implementation process affects implementation outcomes, one will investigate the impact of the initiative on patients and healthcare providers and the final study will identify success factors to support upscaling of the intervention if it is found to be acceptable to whanau and healthcare provides, effective and cost-effective.

\section{ETHICS AND DISSEMINATION}

Trial results will be presented to practices and their patients and funders at appropriate fora, and disseminated widely through peer-reviewed publications and conference presentations. Eligibility for authorship of publications will be based on International Committee of Medical Journal Editors criteria. The results of this study will be highly relevant to health system funders, given the need for yet absence of a comprehensive and effective T2DM management programme for Māori or Pacific people. 


\section{Author affiliations}

${ }^{1}$ Epidemiology \& Biostatistics, University of Auckland, Auckland, New Zealand

${ }^{2}$ Mana Tu, National Hauora Coalition, Auckland, New Zealand

${ }^{3}$ Department of Statistics, The University of Auckland, Auckland, New Zealand

${ }^{4}$ Te Kupenga Hauora Māori, University of Auckland, Auckland, New Zealand

${ }^{5}$ Department of Health Systems, University of Auckland, Auckland, New Zealand

Acknowledgements The Mana Tū programme was founded on the recommendations from the Oranga Ki Tua Advisory Roopu, a Māori-led collaborative, including whānau, clinicians, researchers, health service planners and providers of whānau ora services, who were convened to develop an evidencebased programme for whānau.

Contributors VS wrote the first draft of the manuscript. All authors reviewed and provided feedback on the draft. YJ estimated sample size. VS, MH and YJ designed the trial. $\mathrm{MH}$ is the principal investigator of the study. MH, TS, JR, TT and PC developed the intervention.

Funding This work was supported by the Health Research Council of New Zealand (grant number 16-736).

Disclaimer The Health Research Council of New Zealand (also the sponsor of the trial) had no role or ultimate authority in study design and will have no role or ultimate authority in any of the following: collection, management, analysis and interpretation of data; writing of the report and the decision to submit the report for publication.

Competing interests TS and TT are employees of, and MH is a contractor to, the National Hauora Coalition, a Māori-led Primary Health Organisation. The National Hauora Coalition is the recipient of a grant for the development, implementation and evaluation of the Mana Tū programme (from the Health Research Council of New Zealand, grant number 16-736). The Mana Tū programme is being implemented in the National Hauora Coalition and its associated general practices. The National Hauora Coalition will be the Network hub for the intervention and will employ the Kai Manaaki. All other authors have nothing to disclose.

Patient consent Not required.

Ethics approval Ethical approval for the trial has been obtained from the New Zealand Health and Disability Ethics Committee (17/NTB/249).

Provenance and peer review Not commissioned; externally peer reviewed.

Open access This is an open access article distributed in accordance with the Creative Commons Attribution Non Commercial (CC BY-NC 4.0) license, which permits others to distribute, remix, adapt, build upon this work non-commercially, and license their derivative works on different terms, provided the original work is properly cited, appropriate credit is given, any changes made indicated, and the use is non-commercial. See: http://creativecommons.org/licenses/by-nc/4.0/.

\section{REFERENCES}

1. Harwood M, Tipene-Leach D. Maori and diabetes. In: Robson B, Harris R, eds. Hauora: maori standards of health iv a study of the years 2000-2005. Wellington: Te Ropu Rangahau Hauora a Eru Pomare, 2007.
2. Ministry of Health. New Zealand health survey. annual data explorer. Wellington, 2017.

3. Ministry of HealthMinistry of Health. Tatau Kahukura: māori health chart book. 3rd edn. Wellington, 2015.

4. Health Partners Consulting Group. Metro-Auckland Pacific population health profile. Auckland: Health Partners Consulting Group, 2012.

5. Herman WH, Zimmet P. Type 2 diabetes: an epidemic requiring global attention and urgent action. Diabetes Care 2012;35:943-4.

6. Cram F. Improving Maori access to diabetes health care: literature review. Auckland: Katoa Ltd, 2014.

7. Chan AW, Tetzlaff JM, Altman DG, et al. SPIRIT 2013 statement: defining standard protocol items for clinical trials. Ann Intern Med 2013:158:200-7.

8. King A. Minimum requirements for primary health organisations. Wellington: Ministry of Health, 2001.

9. Best Practice Advocacy Centre New Zealand. Clinical audit. Stepping up treatment in people with poorly controlled diabetes. New Zealand: Best Practice Advocacy Centre New Zealand.

10. Ministry of Health. Ethnicity data protocols for the health and disability sector. Wellington: Ministry of Health, 2004.

11. Salmond C, Crampton P, Atkinson J. NZDep2006 Index of deprivation. Wellington: Department of Public Health, University of Otago, 2007.

12. New Zealand Guidelines Group. Guidance on the management of type 2 diabetes 2011. Wellington: New Zealand Guidelines Group, 2011.

13. Ministry of Health. Cardiovascular disease risk assessment. New Zealand Primary Care handbook 2012. Wellington: Ministry of Health, 2013.

14. New Zealand Guidelines Group. New Zealand primary care handbook. Wellington: New Zealand Guidelines Group, 20122012.

15. Tricco AC, Ivers NM, Grimshaw JM, et al. Effectiveness of quality improvement strategies on the management of diabetes: a systematic review and meta-analysis. Lancet 2012;379:2252-61.

16. Campbell MK, Piaggio G, Elbourne DR, et al. Consort 2010 statement: extension to cluster randomised trials. $B M J$ 2012;345:e5661.

17. Kenealy T, Orr-Walker B, Cutfield R, et al. Does a diabetes annual review make a difference? Diabet Med 2012;29:e217-e222.

18. Pihama L. Tihei mauri ora: honouring our voices. mana wahine as a kaupapa maori theoretical framework. Auckland: University of Auckland, 2001.

19. Smith LT. Decolonizing methodologies: research and indigenous peoples. London, UK: Zed Books, 2012.

20. Schulz KF, Altman DG, Moher D, et al. CONSORT 2010 Statement: Updated guidelines for reporting parallel group randomised trials. $J$ Clin Epidemiol 2010;63:834-40.

21. Hennekens $\mathrm{CH}$, Buring JE. Epidemiology in medicine. Boston: Little, Brown and Company, 1987.

22. Beaglehole R, Bonita R, Kjellstrom T. Basic epidemiology. Geneva: World Health Organization, 2002.

23. Pipi K, Cram F, Hawke R, et al. A research ethic for studying maori and iwi provider success. Social Policy J of New Zealand Te Puna Whakaaro 2004;23:141-53.

24. Kim KB, Kim MT, Lee HB, et al. Community health workers versus nurses as counselors or case managers in a self-help diabetes management program. Am J Public Health 2016;106:1052-8. 